\title{
EFFECT OF REINFORCEMENT ON STRENGTH BEHAVIOR OF HANDMADE JUTE PAPER
}

\author{
H. M. Zakir Hossain ${ }^{1}$, Md.Kamal Uddin ${ }^{1}$, S. M. B. Rahman ${ }^{2}$, Khaled Saifullah ${ }^{3}$ and M. M. Rashid ${ }^{4}$ \\ ${ }^{1}$ Pilot Plant and Processing Division, Bangladesh Jute Research Institute, Dhaka, Bangladesh \\ ${ }^{2}$ Jahangirnagar University, Dhaka, Bangladesh \\ ${ }^{3}$ Mechanical Processing Division Bangladesh Jute Research Institute, Dhaka, Bangladesh \\ ${ }^{4}$ Textile Physics Division, Bangladesh Jute Research Institute, Dhaka, Bangladesh \\ E-mail: engrzakir_bjri@yahoo.com, sfllhkhaled@yahoo.com
}

\begin{abstract}
To produce high strength handmade paper, handmade was reinforced by jute fibre along with/without internal additives (rosin, wax etc.), external additive such as Microcrystalline Cellulose(MCC) suspension. Optimum result was obtained when MCC suspension was applied as external additive along with reinforcement .The strength was increased by $34.69 \%$ due to reinforcement and application of MCC suspension as external additive. The application of rosin along with reinforcement did not play any a significant role in imparting strength and application of wax emulsion hampered the strength of paper.
\end{abstract}

Keywords: Handmade, Jute, Paper, Reinforcement, Strength

\section{Introduction}

The use of Polypropylene (PP) and Polyethylene (PE) as packaging materials has thrown threat to the environment and economy of Bangladesh [1]. Reportedly huge amount of land in Bangladesh are occupied daily by non-degradable polybags which reduces the fertility of land and obstructs the flow of rain water resulting flood in Bangladesh. In this situation, development of a degradable natural packaging material is very important and paper made of cheap jute fibre having high strength and high hydrophobic character can be a solution. Besides this, due to inroads of synthetics, the use of jute as packaging purpose has been decreased [2]. On the other hand, demand of nonwood plant fibres as raw material has been being increased day by day [3-4]. Hence the use of jute could be diversified by using it as the raw material of paper.
It has been reported that when MCC in the form of regenerated cellulose gel is mixed with bleached kraft pulp to produce hand sheet (Hand towel), the mechanical properties (Young Modulus, Tensile \& Tear indices, Folding Endurance) of hand sheet are improved [5]. Swollen starch pulp when used with wood pulp, increase the tensile strength folding endurance of hand sheet as starch pulp had reinforcing effect on inter fibre bonding between wood pulps in the hand sheets. Adding poly-amidiamine epicholohydrin resin (PAE), a wet strength resin, together with swollen starch pulp had a synergistic effect on the increase in folding endurance [6]. The effect of MCC, Carboxymethyl Cellulose (CMC) and mixture MCC and CMC on hydrophobic property of paper produced from jute was studied. It has been observed that the treatment with MCC suspension increased the water repellent property of handmade paper. In case of treatment by mixture of MCC suspension and CMC solution in the ratio of 50:50, the hydrophobic character is usually increased with the increase of MCC concentration in the mixture but the hydrophobic character is decreased with the increase of CMC solution concentration in the mixture [7-8].

It has been reported that application of glutaraldehyde and poly (vinylalcohol) not only improved wet strength of paper but also significantly increased dry strength, tensile energy absorption and folding endurance of treated paper [9].

The effect of fibre reinforcement along with or without sizing material, internal sizing material (rosin, alum, wax emulsion etc) and external (MCC suspension) is yet to be studied. Hence Handmade papers produced from jute were 
treated with various internal sizing materials (rosin alum, wax emulsion) and external sizing material (MCC solution prepared from jute [10]) and raw jute fibres were reinforced to increase the hydrophobic property of paper in this present work.

\section{MATERIALS AND METHODS}

Bangladesh white B (BWB) jute was collected from Mechanical Processing Division of Bangladesh Jute Research Institute and Mennonite Central Committee provided chemicals and laboratory facilities. Ten experiments were performed. Such as: Experiment 01: To make control paper without using any sizing/ reinforcement.

Experiment 02: To make paper without using any sizing but incorporating jute fiber reinforcement
Experiment 03: To make paper without using any sizing but incorporating jute fiber reinforcement and using Micro-crystalline cellulose (MCC) as external additive.

Experiment 04: To make paper without using any sizing but incorporating jute fiber reinforcement and using wax emulsion as external additive.

Experiment 05: To make paper using rosin and alum as internal sizing and incorporating jute fiber reinforcement.

Experiment 06: To make paper using rosin, alum and wax emulsion as internal sizing and incorporating jute fiber reinforcement.

Experiment 07: To make paper using rosin and alum as internal sizing incorporating jute fiber reinforcement as well as using MCC as external sizing.

Table 1 Parameters Used for Pulp Preparation in Different Experiments

\begin{tabular}{|c|c|c|c|c|c|}
\hline \multirow{2}{*}{$\begin{array}{l}\text { Experiment } \\
\text { No. }\end{array}$} & \multicolumn{2}{|c|}{ Atmosphere of Pulp Preparation } & \multirow{2}{*}{$\begin{array}{l}\mathrm{p}^{\mathrm{H}} \text { After } \\
\text { Washing }\end{array}$} & \multirow{2}{*}{$\begin{array}{c}\mathrm{p}^{\mathrm{H}} \text { with } \\
\text { Additives }\end{array}$} & \multirow[t]{2}{*}{ Remarks } \\
\hline & Temperature ${ }^{0} \mathrm{C}$ & $\begin{array}{c}\text { Relative } \\
\text { Humidity (\%) }\end{array}$ & & & \\
\hline 1 & 27.0 & 47 & 7.5 & - & $\begin{array}{c}\text { No additive } \\
\text { used }\end{array}$ \\
\hline 2 & $27.7-32.7$ & $37-46$ & 7 & - & $\begin{array}{l}\text { No additive } \\
\text { used }\end{array}$ \\
\hline 3 & $27.7-32.7$ & $37-46$ & 7 & - & $\begin{array}{c}\text { No additive } \\
\text { used }\end{array}$ \\
\hline 4 & $27.7-32.7$ & $37-46$ & 7 & - & $\begin{array}{c}\text { No additive } \\
\text { used }\end{array}$ \\
\hline 5 & $28.4-28.7$ & $39-50$ & 7.5 & 5.5 & \\
\hline 6 & 31.1-31.5 & $27-33$ & 7.5 & 6.5 & \\
\hline 7 & $28.4-28.7$ & $39-50$ & 7.5 & 5.5 & \\
\hline
\end{tabular}

\subsection{Preparation of fiber sample:}

The fibers were cleaned carefully so that no barks. roots were present. These cleaned fibers were cut to the size of $1.27 \mathrm{~cm}-2.54$ cm ( $1 / 2$-1inch)

\subsection{Preparation of pulp:}

2.2.1 Boiling: $300 \mathrm{~g}$ of cut fibers were taken.18 g sodium hydroxide $(6 \%$ of the arterial) was dissolved in 10 liter of water by boiling. The amount of water was maintained about 10 litre through the whole boiling process. As soon as a water reached the boiling temperature fibers were immersed in the boiling water of a open tube and boiling was continued for 4 hours to remove some impurities as may be soluble in mild alkalis at moderately high temperature as well as to soften and condition the fiber. The boiled fibers were then properly washed by water until the effluent was practically clear. The length of washing time was so adjusted that the fiber/ stock will be as clean as possible with a minimum of fiber loss.

2.2.2 Beating: The washed jute fibers were taken with $70 \mathrm{~kg}$ of fresh water in the open vessel of beater. The fibers were converted into a pulp through cutting and beating in beater for $21 / 4-2 \frac{1}{2}$ hours. The pulp was keep standing 
for 17.25 hours. Then pulp was washed to Maintain pH 7.

2.3 Internal Sizing: The washed pulp had under gone different sizing treatment to increase strength and hydrophobic property of the papers to be produced. Pulp was kept in a open bucket containing 20 Litter of water.

a) $1.5 \%$ Rosin (The most commonly used water repellent compound in stock sizing) soap in form of solution was applied and stirred for 5 minutes by blender. Then (after 30 minuets) 3\% Alum solution was mixed and stirred for another 5 minuets.

b) $1.5 \%$ Rosin soap was applied and stirred for 5 minutes by blender. Then (after 30 minutes) $0.75 \%$ was emulsion was mixed and stirred for another 5 minutes. Then $3 \%$ alum in the from of solution was applied and stirred for 5 minutes. The pulp was kept for 20 minutes after applying internal sizing.

- On the wt. fibre material.

2.3.1Preparation of Rosin size soap: $500 \mathrm{~g}$ of powdered rosin was taken. $50 \mathrm{~g} \mathrm{NaoH}$ ( $10 \%$ of rosin powder) was dissolved in to 1 liter of water by heating .Rosin powder was first converted in to paste by adding some hot caustic soda solution. Rosin soap was prepared by adding the remaining caustic soda solution with Rosin paste and boiled 30 minuets.

2.3.2 Preparation of Rosin Size Solution: $1.5 \%$ (on the wt. of fibre material) i.e. $4.5 \mathrm{gm}$ rosin soap was taken and solution prepared by diluting Rosin soap with hot water.

2.3.3 Preparation of Alum Solution: 3\%( On the wt. fiber material) i.e. 9gm alum was taken. Alum solution was prepared by adding hot water.

2.4 Preparation of wax solution: $0.75 \%$ (On the wt. fiber material) i.e. 2.25g liquid paraffin wax was mixed in water by stirring in a blender. No emulsifier was used.

2.5 Washing of pulp/stuff: The stuff after unloading from beater was kept on a net to allow the liquid to be drained out. The stuff was washed properly by water so that the stuff gets neutral condition.
2.6 Determination of $\mathbf{p}^{\mathbf{H}}$ : the $\mathrm{p}^{\mathrm{H}}$, the indirect measure of the chemical state of the stuff. Sowing the degree of acidity or alkalinity was measured by $\mathrm{p}^{\mathrm{H}}$ paper.

2.7 Lifting: 1 liter of polyamide solution $(0.05 \%$ i.e $7.5 \mathrm{gm}$ polyamide in 15 liter water) was taken in lifting vat containing 20 liter of water. Pulp was suspended uniformly in water of lifting vat. Pulp was lifted from the lifting vat in sheet form or lap form by lifting net and kept on a bed table one after another using pieces of cloth as separators.

2.8 Reinforcement of fiber: Jute fiber filament from carded sliver of BTB jute was collected. These filaments were reinforced between two layers of pulp manually. The distance between fibers (both vertically and horizontally) was kept $2.54 \mathrm{~cm}$.

2.9 Pressing: The pulps in lap form were pressed for 15 minutes by hydraulic press having a capacity of 10 ton to from paper sheet.

2.10 Drying of paper: The produced papers were dried in sunlight.

2.11 Calendaring: The paper sheets got deformed on drying. It was tried to make paper sheet flat by calendaring.

\subsection{External sizing:}

a) $0.5 \%$ MCC suspension (10g MCC in 2000cc water) was prepared. Paper samples were soaked in the suspension of MCC for 1 minute. Then samples were dried in sunlight.

b) $0.5 \%$ wax emulsion (i.e. $5 \mathrm{~g}$ in $100 \mathrm{cc}$ water) was prepared. Paper samples were soaked in wax exclusion for 1 minute. Then samples were dried in sunlight.

\subsection{Test of the Properties:}

The physical properties of paper like thickness, GSM, tearing strength were measured for each experimental group in the standard temperature and humidity (Temp $=23 \pm 1^{\circ} \mathrm{C}, \mathrm{RH}=50 \pm 2 \%$ ) for paper as per TAPPI standard test methods ${ }^{11-12}$ after conditioning for 3days according to (T402 gm-93). For each experiment, samples were cut from different sheet (one from each sheet)

2.13.1 Thickness: 10 samples $(10 \mathrm{~cm} \times 10 \mathrm{~cm})$ for each experiment were taken. Thickness was measured by vernire calipers according to TAPPI T441om-97. Five reading were taken from each sample and results were recorded in mm. 
2.13.2 GSM: 10 Samples $(10 \mathrm{~cm} \times 10 \mathrm{~cm})$ for each experiment were taken. The weight of the samples was taken by electronic balance and these were multiplied by 100 to get the weight of the sample in Grams per Square Meter (GSM). (Beside this method GSM could be measured according to TAPPI T410 om-98 and results were as $\mathrm{g} / \mathrm{m}^{2}$ Which indicates the was unit area of paper on a sector scale when a sample of $10 \mathrm{~cm} \times 10 \mathrm{~cm}$ size is suspended from a hook fixed to the pointer arm).

2.13.3 Tearing strength: 10 samples (7.6 $\mathrm{cm} \times 6.3 \mathrm{~cm}$ ) were taken. Tearing strength in gm-f was measured according to TAPPI. T414 om-98. Test specimens were cut accurately in each principal direction of the paper about $7.6 \mathrm{~cm}$ in length by exactly 6.3 $\mathrm{cm}$ in width, with slit to be cut $20 \mathrm{~mm}$ in length, leaving exactly $43 \mathrm{~mm}$ between the end of the slit and the edge of the specimen. The machine was leveled and adjusted before each set of test. The pendulum was positioned to test position. Test specimen was placed mid-way in the clamp with its upper edge parallel to the tops of the jaws and the handle was pushed downward to make the slit. The scale reading was found between 20 and 60 when single sheet was torn. Five reading were recorded for each principal direction of the paper (i.e. vertical and horizontal). The readings were rejected where tear line deviates more then 10 $\mathrm{mm}$ from the line of initial slit.

The tearing force and tearing index could be obtained by the following formula:

For the standard 1600-gf instrument with 0-100 scale.

Average tearing force, gf = $\left(\frac{16 \times \text { average scale reading }}{\text { Number of plies }}\right)$

Average tearing force, $\mathrm{mN}=$ $\left(\frac{16 \times 9.81 \times \text { average scale reading }}{\text { Number of plies }}\right)$

Tearing Index $=\mathrm{E} / \mathrm{w}$

Where, $\mathrm{E}=$ The force in $\mathrm{mN}$ to tear a single ply $\mathrm{W}=$ Grammage in $\mathrm{g} / \mathrm{m}^{2}$ (Oven dry wt.)

\section{Results and Discussions}

Table 2 Test results of hand made papers from Jute fibers

\begin{tabular}{|c|c|c|c|c|c|c|c|c|c|c|}
\hline \multirow{2}{*}{$\begin{array}{l}\text { Exp } \\
\text { No }\end{array}$} & \multicolumn{4}{|c|}{ Thickness(mm) } & \multicolumn{4}{|c|}{$\mathrm{GSM}\left(\mathrm{Gm} / \mathrm{m}^{2}\right)$} & \multicolumn{2}{|c|}{ Tearing Strength Test } \\
\hline & Mean & $\begin{array}{l}\text { CVw } \\
(\%)\end{array}$ & Mean & $\begin{array}{l}\text { CVb } \\
(\%)\end{array}$ & Mean & $\begin{array}{l}\text { CVb } \\
(\%)\end{array}$ & Mean & $\begin{array}{l}\text { CVb } \\
(\%)\end{array}$ & $\begin{array}{l}\text { Tear Index } \\
\mathrm{mN} \cdot \mathrm{m}^{2} / \mathrm{Gm}\end{array}$ & $\begin{array}{c}\text { Strength } \\
\text { Increased } \\
\text { (\%) }\end{array}$ \\
\hline 1 & 0.28 & 11.16 & & & 175.8 & 09.84 & & & 33.93 & - \\
\hline 2 & 0.37 & 18.82 & & & 186.3 & 08.57 & & & 42.55 & 25.41 \\
\hline 3 & 0.34 & 12.16 & & & 190.6 & 10.66 & & & 45.70 & 34.69 \\
\hline 4 & 0.30 & 18.97 & 0.33 & 13.41 & 201.5 & 11.16 & 188.86 & 7.88 & 32.95 & -2.89 \\
\hline 5 & 0.35 & 14.29 & & & 202.9 & 16.97 & & & 35.43 & 04.42 \\
\hline 6 & 0.27 & 11.39 & & & 163.6 & 06.27 & & & 34.16 & 00.68 \\
\hline 7 & 0.38 & 22.38 & & & 201.3 & 15.00 & & & 36.96 & 08.93 \\
\hline
\end{tabular}

$\mathrm{CV}_{\mathrm{w}}=\mathrm{Co}-$ efficient of Variation within experiment

$\mathrm{CV}_{\mathrm{b}}=$ Co-efficient of Variation between experiments

Tearing Index $=$ Tearing Force $(\mathrm{mN}) / \mathrm{GSM}$ $\left(\mathrm{Gm} / \mathrm{M}^{2}\right)$

Experiment 01: Control

Experiment 02: Fiber Reinforced

Experiment 03: Fiber Reinforced + MCC as external additive
Experiment- 04 : Fiber Reinforced + Wax as external additive

Experiment- 05: Rosin + Alum + Fiber Reinforcement

Experiment- 06: Rosin+ Alum+Wax as internal additive+Fiber Reinforcement

Experiment - 07: Rosin + Alum+ Fiber

Reinforcement+ MCC as external additive

From result table it was observed that the mean thickness and GSM of paper were $0.33 \mathrm{~mm}$ and 188.86 respectively. The mean thickness of paper 
varied a lot as the CV\% of paper thickness (within experiments) were $11.16 \%-22.38 \%$ and the CV\% (among experiment) was $13.41 \%$. The mean GSM of paper also varied as the CV\% were $6.27 \%-16.97 \%$ (within experiment) and that among experiment was $7.88 \%$. The variation in thickness and GSM was due to lack of uniformity in lifting.

It was found that the paper strength was increased due to reinforcement of jute fibre in all but one (experiment no. 4) case when paper was treated by wax emulsion. But the increase in strength due to reinforcement was unexpectedly low due to lack of cohesion between reinforcing fiber and pulp as well as long distance between the reinforcing fibers. The application of rosin along with reinforcement did not play any a significant role in imparting strength to the paper as increase in strength was very low (experiment no 5,6 \&7)

The strength of paper was increased by 25.41 $\%$ when it was reinforced by jute fibre (experiment no. 2) and the strength was enhanced up to $34.69 \%$ (experiment no.3) when the paper was treated by MCC suspension as external additive along reinforcement.

\section{Conclusion}

The use of jute has been declined in the last few decades due to inroads of synthetic fibres. For this reason scientists are trying to develop diversified jute products. Handmade jute paper is bio-degradable and environmental friendly. It may be a potential substitute of polypropylene and polyethylene for packaging materials.

\section{References}

[1] Muhmmad Serazul Haque, "Manufacturing and marketing of cheap jute bag as substitute of polyethylene" keynote papr presented in the seminar titled "To encourage the use of cheap jute bag as substitute of ployethylene forpreventing environment pollution" held on January 26,2002, Dhaka,Bangladesh

[2] Mohammad Ali "Modification of jute fibre and faricsBangladesh" Vol.11 No.9\&10 p-20

[3] Atchison, J.E, "Worldwide capacities of non-wood plant fibre pulping increasing faster than wood pulping capacities”.TAPPI Nonwood plant fibre Pulping Progress Report, 1990, No.19, pp.1-7

[4] Chandra, Mudit. "Use of nonwood plant fibres for Pulp and Paper industry : Focus on China",masters dissertation, Virgina Polytechnic Institute and State University, Virgina, USA, 1997 '.

[5] Isogai, Akira "Properties of Hand sheets Containing Cellulose Regenerated from Cellulose/Aquous $\mathrm{NaOH}$ solution”, "Journal of the Society of Fiber Science and Technology, Japan”. Vol. 53 No.3, March 1997, (Abstracted in Textile Research Journal, USA. March1997)

[6] Suzuki, Takashi; Isogai, Akira; Onabe, F "Properties of Hand sheets Containing Starch Pulp Prepared with Various Wet-End Additives", "Journal of the Society of Fiber Science and Technology, Japan” Vol.53 No.10, March 1997, (Abstracted in Textile Research Journal, USA. October-1997).

[7] Hossain,H.M.Zakir;Mollah,M.E.;Rahman,SMB;Rashid and M M;Abdullah, "Application of Micro-crystalline cellulose to Increase the Hydrophobic Property of Paper Produced from Jute Waste”, "Bangladesh Journal of Jute and Fibre Research”Vol.16 No.1-2, 2006,pp.12-17

[8] Hossain,H.M.Zakir,Mollah,M.E.;Rahman,SMB;Matin, Nilufar and Shahidullah,Md. "Modification of Handmade Paper From Jute by CMC in mixture with MCC suspension for Increasing Hydrophobic Property", "Bangladesh Journal of Jute and Fibre Research” Vol.16 No.1-2,2006,pp.18-23.

[9] Abdullah; Khan; Salam; "Preparation of Microcrystalline Cellulose from Jute", "Bangladesh Journal of Jute and Fibre Research", No.3, 1978, pp.39-43 